\title{
Making sense of what enables and what constrains adaptation to climate change
}

\author{
Tim Lynam $^{\text {a }}$ \\ ${ }^{a}$ Social and Economic Science Program, CSIRO, Townsville, Australia. \\ Email:Tim.Lynam@csiro.au
}

\begin{abstract}
As a first step in responding to new phenomena such as climate change people need to make sense of these new phenomena. Social representations theory suggests that people first anchor new phenomena to what they already know and then through processes of social interaction such as conversation or engagement with public media, people objectify this anchored representation in the form of an image, metaphor or symbol. This objectification makes the phenomenon familiar and part of common sense. As such the objectified phenomenon is added as a new social object to the group's world. The social representations form an important underpinning feature of how people respond to phenomena such as climate change.
\end{abstract}

The process of making sense of phenomena such as climate change is thus very much a social process and we need to understand how this process occurs in order to effectively model people's responses. In a similar vein, to understand how people respond to climate change we need to make sense of how they make sense of the situations they face; what enables and what constrains their ability to adapt to climate change. These enabling and constraining factors need to be understood as objects within the social world. Making sense of new phenomena is a highly complex process. As society grapples with making sense of climate change and having to adapt to climate change it is very difficult to predict what social objects will emerge and become objectified. This is a truly difficult situation to model.

To build models of the social dimensions of climate change we will need as a first step, to be able to model this highly complex sense making process through which groups within, and society as a whole come to terms with climate change and what enables them to adapt to climate change. In this paper we describe some preliminary results from our investigations of sense making in relation to climate change and climate change adaptation among different social groups. Our data compromise almost 1000 narrative fragments of people's personal experiences of what enables and what constrains adaptation to climate change. We present some preliminary findings of patterns in how society in Australia and Canada, are making sense of adapting to climate change.

Respondents in our survey revealed characteristics of their narratives and their own relationship to their narrative as well as a number of characteristics of themselves. We present preliminary results of mixed methods analysis of word association and narrative fragment data on what people think of in relation to climate change and in which factors help and which hinder adaptation to climate change. An important conclusion emerging from our preliminary analyses of the data was that there was no one consensual representation either of climate change or the factors that enable or constrain adaptation to climate change. The paper concludes by identifying some opportunities and difficulties that our analyses present for modelling the social dimensions of climate change.

Keywords: sensemaking, adaptation, climate change, word associations, modelling 


\section{INTRODUCTION}

Adaptation is not new; people as individuals, as groups and as societies have been adapting to changing circumstances for millennia. Climate change however, is a new phenomenon that current human society has not experienced. Global scale environmental changes have been causally associated with significant societal change (Zhang et al. 2007, Haberle and Lusty 2000, Diamond 2005). If current society sought to invest now to increase the capacity of society to adapt to the changes that climate change will bring where should it make those investments? Should we be developing better information systems to enable society to better predict climate impacts? Would we be better off building climate proof infrastructure to support future societies? Each of the possible investment options has advantages and disadvantages and our ability to answer now what is the best investment portfolio is somewhat limited by our ability to predict how people will respond to the situations of the future. Can we predict future pathways of human behaviour sufficiently well to provide insight for current investment decisions in building capacity for future adaptation? Philip Tetlock demonstrated that human experts do not predict the outcome of large scale human endeavours with much accuracy at all; experts in the field generally predicted worse than statistical algorithms (where the tested algorithms ranged from simple base-rate extrapolations to generalized autoregressive distributed lag models). The best of the models could explain on average $47 \%$ of the observed variance. Human experts "were hard-pressed to predict more than 20 percent of the total variability in outcomes..."(Tetlock 2005).

As a first step in responding to new phenomena such as climate change people need to make sense of these new phenomena. Social representations theory suggests that people first anchor new phenomena to what they already know and then through processes of social interaction such as conversation or engagement with public media, people objectify this anchored representation in the form of an image, metaphor or symbol. This objectification makes the phenomenon familiar and part of common sense. As such the objectified phenomenon is added as a new social object to the group's world (Wagner et al. 1999). The social representations form an important underpinning feature of how people respond to phenomena such as climate change.

The process of making sense of phenomena such as climate change is thus very much a social process and we need to understand how this process occurs in order to effectively model people's responses. In a similar vein, to understand how people respond to climate change we need to make sense of how they make sense of the situations they face; what enables and what constrains their ability to adapt to climate change. These enabling and constraining factors need to be understood as objects within the social world. Making sense of new phenomena is a highly complex process as (Snowden and Boone 2007, Kurtz and Snowden 2003) have pointed out; our assumptions of discernible and repeatable cause effect relationships may not hold. As society grapples with making sense of having to adapt to climate change it is very difficult to predict what social objects will emerge and become objectified. This is a truly difficult situation to model.

In this paper we explore a simple aspect of human interaction with new phenomena; how society makes sense of the phenomena. We discuss this process with reference to the concept of climate change, modelling the manner in which a society extracts sense from a complex set of interrelated concepts. We show that this sense-making process has implications for climate change adaptation and mitigation. We start by briefly exploring how people across Australia and parts of Canada anchor and objectify representations of climate change. We then explore how they represent what enables and what constrains their adaptation to climate change. We then pay particular attention to information and discuss how people's conceptions of the importance of information vary as we explore the different perspectives they adopt. We conclude by identifying the implications of our analyses for modelling human adaptation and also make recommendations for how we might proceed with modelling, from the perspective of policy evaluation.

\section{METHODS}

A survey instrument was designed and administered as an online instrument to three populations of potential respondents; attendees at a climate adaptation conference in July 2010; a Victorian government department

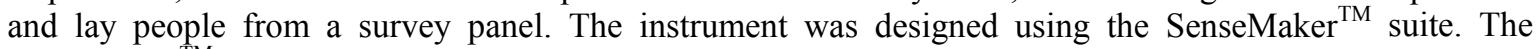
SenseMaker ${ }^{\mathrm{TM}}$ survey instrument was designed to capture individual responses to questions as to what enabled and what constrained adaptation to climate change. The instrument was designed to enable capture and subsequent analysis of both qualitative and quantitative responses from individuals who were presented with the same situation or context in which to frame their responses. 
Two slightly different instruments were used with respondents being randomly assigned to one or the other of them with equal probability. The only difference between the instruments was that one of them included three word association questions as the first task and the other instrument had no word association questions. The analyses presented in this paper were based on data from the completed instruments with word association questions $(\mathrm{n}=540)$.

The instrument comprised five tasks but only the results of the first are analysed and presented here. In the first task (a word association task) respondents were first asked to list the first words that came to mind when they thought of climate change, then asked to write down the most important things that help people deal with climate change and lastly, to write down the most important factors that hinder people from dealing with climate change. The word association data were analysed using QDAMiner to identify relative frequencies of words (and concepts) across individuals and the co-occurrence of terms in each response. Similarity matrices were generated from term co-occurrence matrices using Jaccard's coefficient (Salton 1989). The similarity matrices were used in non-metric multi-dimensional scaling (MDS) analyses with the lowest stress (i.e. best fit) result selected from 1000 random initial configurations.

\section{RESULTS}

\subsection{What is climate change?}

What comes to mind when you think about climate change? Respondents in the survey most commonly associated climate change with "weather", with "change", with the "sea", with "global", with "warming", with "climate" and climatic events (Figure 1). The multi-dimensional scaling plot of Figure 1 shows the tf-idf weight (Salton, Fox and Wu 1983) reflecting the

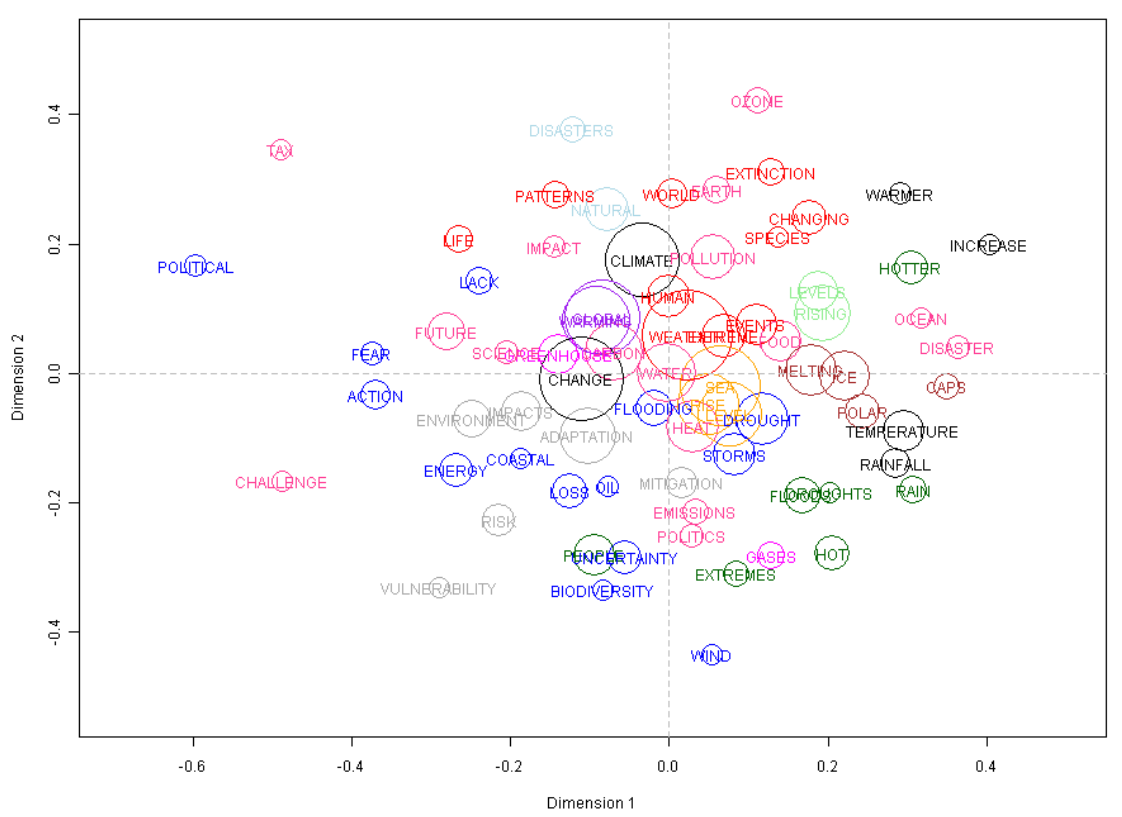

Figure 1. Multi-dimensional scaling (MDS) plot of within sentence word associations from the question "Please write down the first words that come to mind when you think about climate change". Stress $=0.235$. Colours reflect different clusters identified through hierarchical cluster analysis. relative importance of terms across respondents (larger size of circle $=$ greater weight). The proximity of words to each other in respondents' word association tasks are represented by distance from each other in the plot. We use colour in the plot to highlight groups of words that were identified using hierarchical cluster analysis.

In the centre of the plot are the dominant terms and their proximity to each other indicates a high degree of cooccurrence in the word association task. In the top left quadrant of the plot are clustered terms associated with climate change itself ("global warming", "carbon", and "greenhouse"). The top right quadrant includes many terms associated with the weather ("extreme events", "hotter", and "warmer") as well as some important impacts ("extinction", "food" and "rising" sea "levels" associated with "melting ice"). In the bottom right quadrant are many terms associated with the climate ("hot", "heat", "rainfall" and "rain") as well as the impacts of these ("floods", "droughts" and "storms"). In the bottom left quadrant the dominant terms are "change", "adaptation", "environment", "impacts", "people" and "flooding" but also terms associated with loss ("loss", "risk" and "vulnerability") and with energy. The plot also yields some 
interesting observations around closely related terms; "fear" and "action" for example or "people" and "uncertainty".

\subsection{Factors that helped people adapt to climate change}

What did respondents think were most important things that help people deal with climate change? "Information", "education", "knowledge" and "understanding" were dominant concepts in people's expressions of what helped them deal with climate change (Figure 2). Interestingly "education" was closely associated with "information" whilst "knowledge" was more closely associated with "understanding". In the top left quadrant of the plot we find terms associated with "information" including "science", "clear",

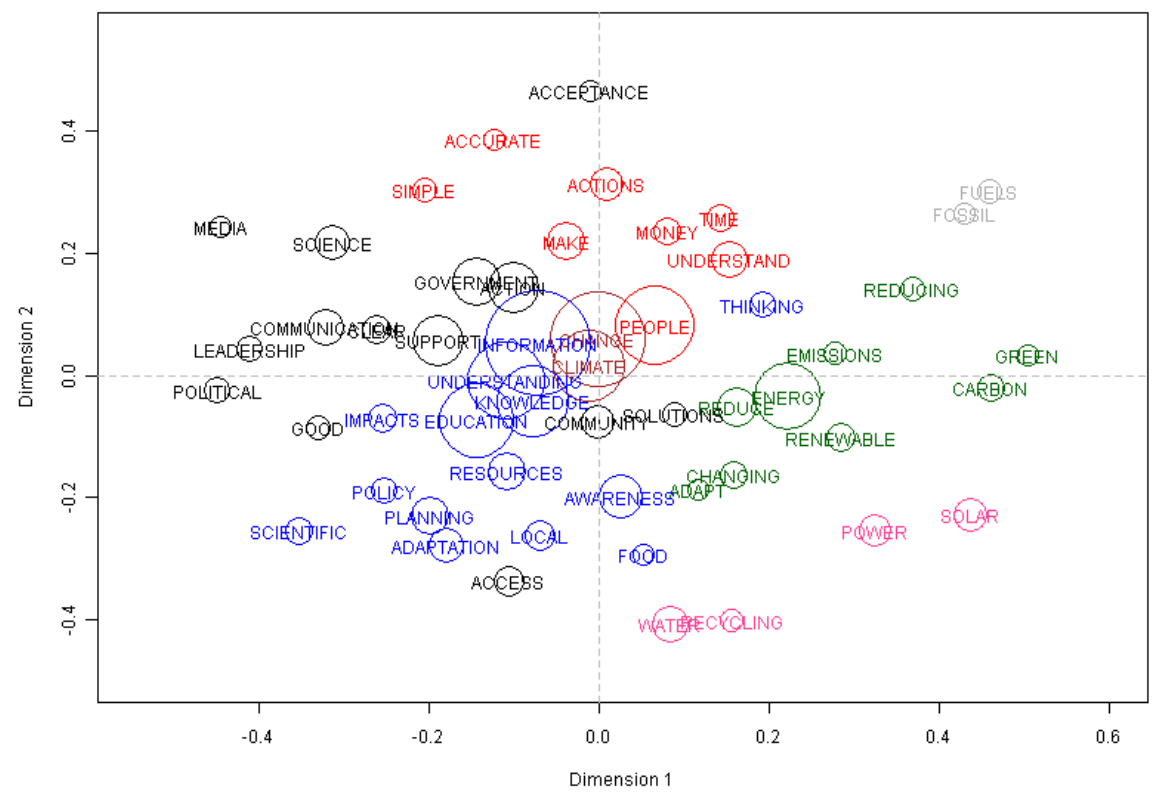

Figure 2. MDS plot of within sentence word associations for "Please write the most important things that help people deal with climate change". Stress $=0.196$. Colours reflect different clusters identified through hierarchical cluster analysis.

"simple", "accurate", "government" and "communication". We also find an interesting group of terms to do with "leadership", "government", "support" and "action". In the top right quadrant of the plot are found terms associated with "people" taking mitigating actions ("reducing", "fossil fuels", "emissions" and "green carbon"). Also notable in this quadrant are the terms "money", "time" and "understand"; suggesting some of the important requirements for

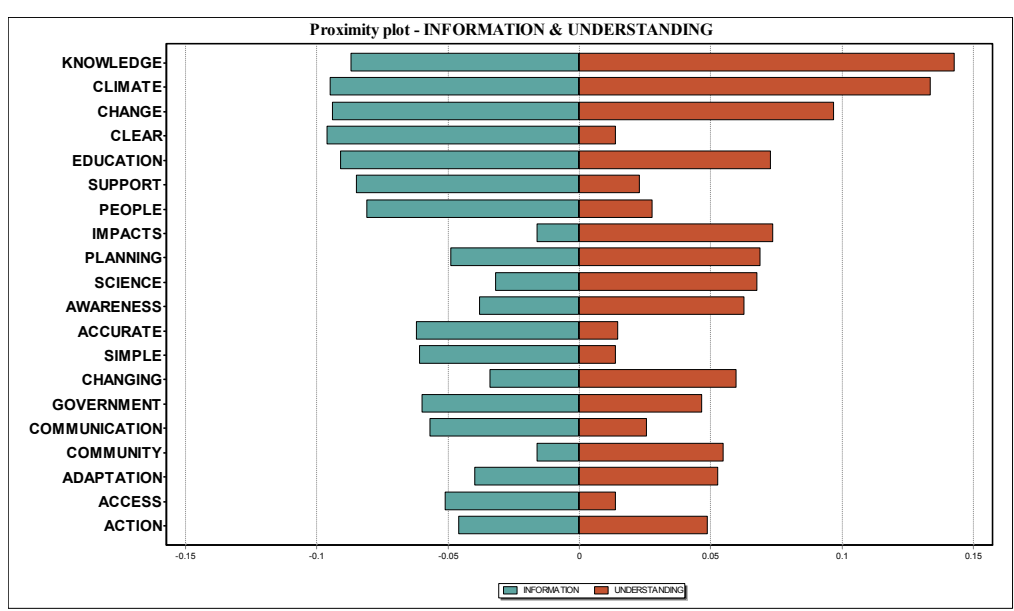

Figure 3. Information and understanding proximities to other top 20 concepts from the word association task of things that help people deal with climate change. X-axis values reflect the probabilities of cooccurrence with the use of negative terms on the left simply enabling the separation of the two terms "information" and "understanding". adaptation. In the bottom right quadrant are terms associated with more environmentally benign adaptations or technologies ("renewable", "energy", "solar", "power" and "water" "recycling"). The bottom left quadrant can perhaps be thought of as representing terms to do with "understanding", including "knowledge", "education", "planning and "policy".

Examination of the co-occurrences of some of the key terms from Figure 2 yielded some interesting insights (Figure 3); we have already identified how "knowledge" and "understanding" were frequently associated whilst "education" and "information" were frequently associated. The associations suggest that people seek "accurate", "clear" and "simple" information on climate change and 
want to understand the impacts. Also noteworthy was the association between "government" and "information" and the association between "community" and "understanding". Interestingly, the term "adaptation" was more often associated with "understanding" than with "information".

We also examined the likelihood of words co-occurring with each of three groups who might be deemed to be associated with responsibility; government, people and science. In many respondents' minds government was clearly associated with "support", "change" and "leadership". Surprising for us were the words most often associated with the term "action"; "community" was by far the dominant word associated with "action".

\subsection{Factors that hindered adaptation to climate change}

In contrast to the factors helping people to adapt, there did not appear to be as clear a representation for the factors that hindered adaptation. For the most part, a larger number of items were identified by fewer people. A "lack" of "understanding", "information", "knowledge" and "resources" were clearly identified as was "cost" and "fear". A lack of "political leadership" coupled with "short term political time horizons" was frequently noted. Interesting was the association between "misinformation", the "media" and "science". Also interesting was the association between "political" and "science"; "science" was often seen as being "political".

Looking at the words most frequently in close proximity to the dominant term in the word association frequencies, "lack" and "information" were dominant, with "knowledge" and "understanding" following close behind. A "lack" of "change", "ignorance" and a "lack" of "leadership" were interesting indicators of what people see as constraining their ability to adapt.

\section{DISCUSSION}

In discussing the presented results I will

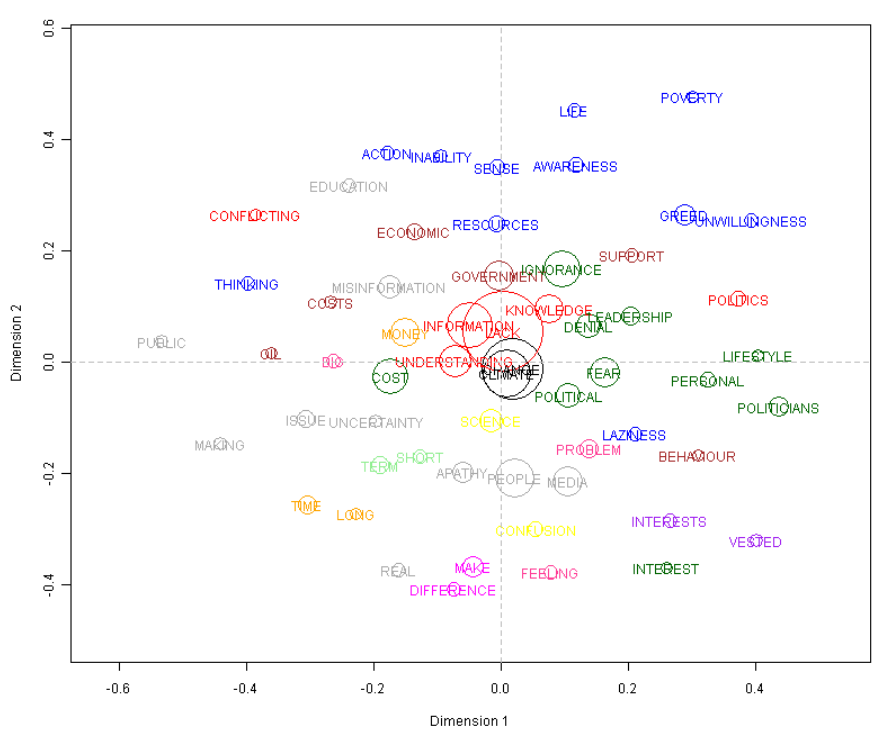

Figure 4. MDS plot of word association "Please write the most important things that hinder people from dealing with climate change". Stress $=0.23$. Colours reflect different clusters identified through hierarchical cluster analysis.

focus on the term "information" as this was a dominant factor that people identified across both what enables and what hinders adaptation to climate change. The term is also useful in exploring the difficulties associated with modelling sense making (Figure 2, Figure 4). What we may deduce from these results is that people identify "information" as helping adaptation and the "lack" of "information" as constraining adaptation. In addition we can see that people want "clear", "simple", "accurate" and "trusted" "information" and see

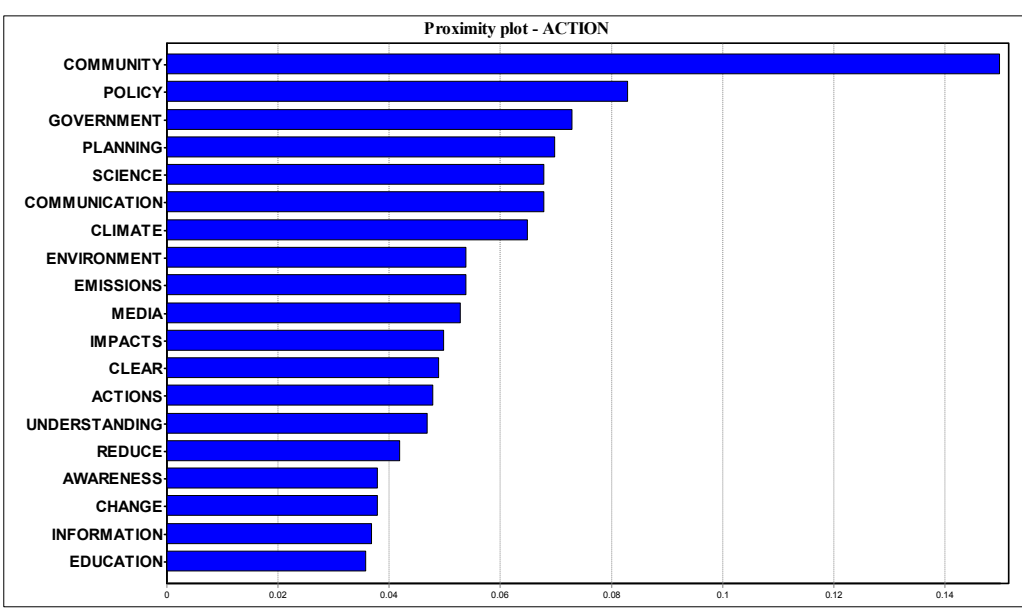

Figure 5. Proximity plot of associations with the word "action" from factors that help adaptation. Within sentence associations. the "government" as an 
important source of this "information" (Figure 2). People want "information" on the nature of the problem (e.g. "climate" and "weather") on possible solutions ("water recycling", "solar power") and to support "planning" and "behavioural change". Looking at this data the clear conclusion would be to provide people with what they want (clear, simple and accurate information, from a source they can trust on the nature of the problem and the solutions they need) and thus better enable them to adapt.

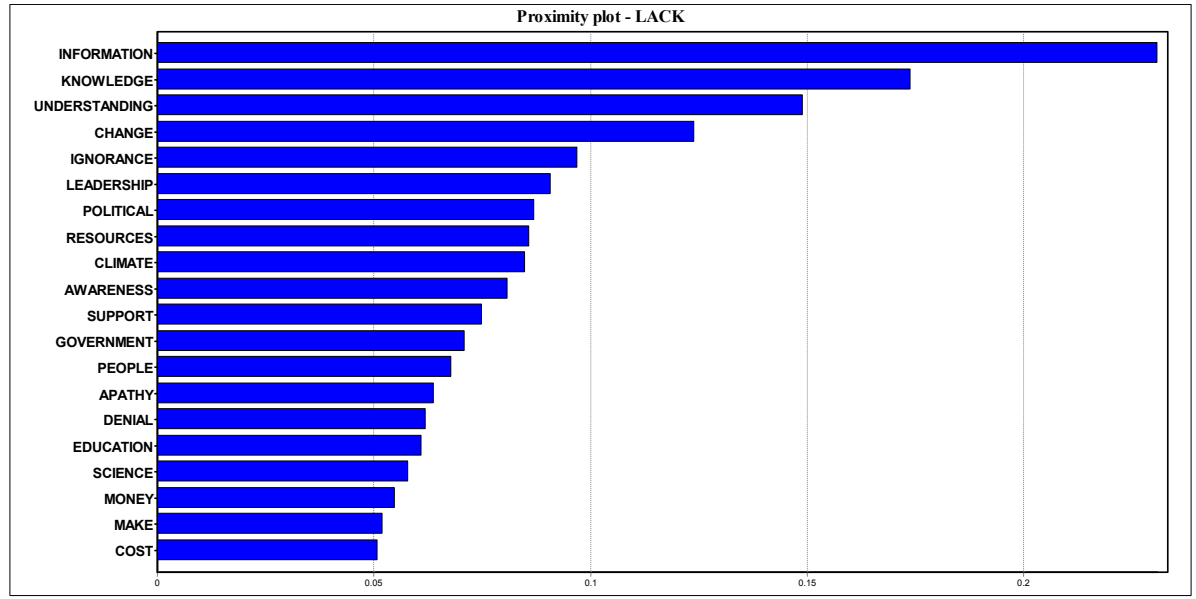

Figure 6. Proximity plot of the term "Lack" in what factors hinder people from adapting to climate change. Association within the same sentence.
Unfortunately this story is not quite as clear as it first appears. Apart from asking people to list the things that helped or hindered adaptation we also asked them to tell of their experiences of adaptation and then asked them to identify the strength of certain factors in their related experiences. What was clear from this second analysis was that a large number of the people who responded felt that information had been overwhelming in the experiences they related. Thus whilst the logical response from the word association data might have been to provide more clear, simple and accurate information, the analysis of people's experiences suggests that response may in fact add to people feeling overwhelmed and hence unable to adapt! This observation highlights the need to adopt multiple perspectives (including analytical perspectives) in seeking to understand human behaviour!

A second thing we need to recognise is the distinction that is apparent in peoples' representations between "information" on the one hand and "understanding" on the other. People associated "action" with both themselves and their communities and "action" is more often associated with "understanding" than with "information" (Figure 5). Interestingly "understanding" is more often associated with the terms "knowledge", "science" and "community" than is "information" and importantly for our discussion "understanding" is more frequently associated with the term "adaptation" than is "information" (Figure 3). These results suggest that people ascribe different meanings to the words "information" and "understanding" and whilst the two are related their use implies different things; people want to understand the impacts of climate change and this understanding helps them to change and adapt. People also want to be educated about the climate and climate change through access to clear, simple and accurate information.

Who provides information or understanding is also a key factor in the representations that people form about adaptation to climate change. The term "government" was most often associated with "support", "leadership", "education" and "information" whilst the term "science" was most often associated with "information", "communication", "action" and "clear". The term "people" was most often associated with "understand" and "understanding", "impact", "community" and "climate". Thus respondents appeared to associate different roles to different social groups.

The data presented in the MDS plots (Figure 1, Figure 2, Figure 4) reflect an emerging and possibly highly volatile set of meanings. How society comes to terms with climate change and adaptation and how these meaning clusters evolve have important implications for how society engages with climate change. It also means that predicting these outcomes with any sense of reliability becomes exceedingly difficult. Our results suggest in fact that the two unordered domains of Snowden and Boone's (2007) Cynefin framework, the complex and chaotic domains, present us with situations in which predictive modelling has little if any utility. Sense making appears to be highly complex and hence calls for alternative approaches to modelling; approaches that can ready accommodate the emergent eddying of meaning. I suggest in fact that modelling in these domains is targeted at sense making; identifying emergent patterns of meaning and exploring their likely implication. Modelling that seeks to identify a) what clusters of meaning might emerge; b) which 
clusters may come to dominate the social meaning; and c) what are the implications (from a policy perspective) of these dominant clusters? Ideally however, this process would seek to include those with the power to influence social outcomes are involved in the sense making.

People make sense using what they already know; anchoring to what is known (Wagner et al. 1999) as well as using their existing knowledge structures to filter information so that they only hear or see what is pertinent to what they already believe (Jones et al. 2011). This is as true for those interpreting the data as for those who are making sense of the phenomenon that we are interested in. We thus need to develop and use modelling processes with key social groups that enable them to make sense of data but also compel them to question their pre-existing assumptions and sense making frameworks. It needs to be stressed that in relation to the unordered domains the modelling process being advocated is not to construct statistical, dynamical, agent based or mathematical models with those needing to make sense and act on their sense making. These are likely to be too constraining and more likely to compel users to see patterns that are built into the models than facilitate processes of examining data from multiple perspectives to identify patterns that emerge when each of these perspectives is adopted. On the contrary the modelling process being advocated here is one through which we seek to make meaning from complex systems, using tools that enable us to take multiple perspectives and where the sense maker and his or her beliefs are recognised as being an actor in the system being modelled and as likely as any to adopt and hold dear a particular perspective.

\section{ACKNOWLEDGEMENTS}

This research was funded by CSIRO's Climate Adaptation Flagship. I am grateful to Kirsty Kitto, Peter Bruza and Fabio Boschetti for their insightful suggestions and comments on an earlier draft.

\section{REFERENCES CITED}

Diamond, J. 2005. Collapse: How societies choose to fail or succeed. New York: Viking.

Haberle, S. G. \& A. C. Lusty (2000) Can Climate Influence Cultural Development? A View through Time. Environment and History, 6, 349-369.

Jones, N. A., H. Ross, T. Lynam, P. Perez \& A. Leitch (2011) Mental Models: An Interdisciplinary Synthesis of Theory and Methods. Ecology and Society, 16, 46.

Kurtz, C. F. \& D. J. Snowden (2003) The new dynamics of strategy: Sense-making in a complex and complicated world. IBM Systems Journal, 42, 462-483.

Salton, G. 1989. Automatic Text Processing. Reading, Mass.: Addison-Wesley.

Salton, G., E. A. Fox \& H. Wu (1983) Extended Boolean information retrieval. Communications of the ACM, $26,1022-1036$.

Snowden, D. \& M. E. Boone (2007) A leader's framework for decision-making. Harvard Business Review, November, 1-9.

Tetlock, P. E. 2005. Expert political judgement: How good is it? How can we know? Princeton: Princeton University Press.

Wagner, W., G. Duveen, R. Farr, S. Jovchelovitch, F. Lorenzi-Cioldi, I. Marková \& D. Rose (1999) Theory and Method of Social Representations. Asian Journal of Social Psychology, 2, 95-125.

Zhang, D. D., P. Brecke, H. F. Lee, Y.-Q. He \& J. Zhang (2007) Global climate change, war, and population decline in recent human history. Proceedings of the National Academy of Sciences, 104, 1921419219. 